\title{
RESEARCH ON THE DIRECT CARBON EMISSION FORECAST OF CHINA'S PROVINCIAL RESIDENTS BASED ON NEURAL NETWORK
}

\author{
Tianning Zhang, Boyan Zhou, Shenghui Zhou, Weiyang Yan* \\ College of Environment and Planning, Henan University, Kaifeng 475004, Henan, China - yanwy9991@163.com
}

KEY WORDS: Global climate change, Residents' carbon emissions, Elman, Neural network, Forecast, China

\begin{abstract}
:
Global climate change, which mainly effected by human carbon emissions, would affect the regional economic, natural ecological en vironment, social development and food security in the near future. It's particularly important to make accurate predictions of carbon emissions based on current carbon emissions. This paper accounted out the direct consumption of carbon emissions data from 1995 to 2014 about 30 provinces (the data of Tibet, Hong Kong, Macao and Taiwan is missing) and the whole of China. And it selected the optimal models from BP, RBF and Elman neural network for direct carbon emission prediction, what aim was to select the optimal prediction method and explore the possibility of reaching the peak of residents direct carbon emissions of China in 2030 . Research shows that: 1) Residents' direct carbon emissions per capita of all provinces showed an upward trend in 20 years. 2) The accuracy of the prediction results by Elman neural network model is higher than others and more suitable for carbon emission data projections. 3) With the situation of residents' direct carbon emissions free development, the direct carbon emissions will show a fast to slow upward trend in the next few years and began to flatten after 2020, and the direct carbon emissions of per capita will reach the peak in 2032. This is also confirmed that China is expected to reach its peak in carbon emissions by 2030 in theory.
\end{abstract}

\section{INTRODUCTION}

Global climate change is closely related to human carbon emissions and has become one of the most important issues in today's society (Qin, 2006). The global climate system is extremely complex, and the factors contributing to global climate change are also complex and diverse (Lean et al., 2008), the fourth assessment report (AR4) released by the Intergovernmental Panel on Climate Change (IPCC) attributed the cause of global warming to greenhouse gas emissions from human activities (Wang, 2010; Fang et al., 2011), and the scientific nature of the conclusion was further confirmed in the fifth assessment report. This conclusion serves as a consensus of governments on climate change issues and provides an important theoretical basis for countries to respond to global changes (Qin, 2016). Therefore, research on the emission reduction of greenhouse gases such as carbon dioxide has become the main content of countries around the world in their efforts to curb global warming trends.

In order to effectively control the emission of greenhouse gases such as carbon dioxide, there is a fierce game between developed countries and developing countries around the obligation of carbon emission reduction. As a major country responsible for the living environment of human beings, China has the responsibility and obligation to actively undertake emission reduction obligations in accordance with its own capabilities while ensuring economic development. On November 30, 2015, President Xi delivered a speech at the opening ceremony of the Paris Climate Change Conference in Paris, France, and promised that China will reach its carbon emission peak around 2030.

China is in a stage of rapid development, rapid urbanization and industrialization, and the direct energy consumption carbon emissions have become two major parts of China's greenhouse gas emissions, related research shows that the residents' consumption carbon emissions of developed countries are increasing, and some have exceeded the carbon emissions in industrial sector (Shui et al., 2005; Druckman et al., 2009), so the control of residents' carbon emissions will be a key research area for carbon emissions reduction. However, the prediction of carbon emissions is particularly important to make a scientific and fair carbon emission plan. At present, the research on carbon emission prediction mostly adopts the method of decomposition and modeling of carbon emission influencing factors (Shi, 2003; Zhu et al., 2009; Qu et al., 2010; Zhao et al., 2013; Huang et al. 2016), but because of different scholars selected different influencing factors and modeling methods, the differences in the prediction results are relatively large, and there are relatively few researches on the direct residents' carbon emissions.

Relying on its unique network structure and data processing characteristics, the artificial neural network model has been widely used in engineering control, natural ecological environment, health care, financial economy, urban intelligent management, and geosciences and other related prediction field (Luo et al., 2013; Sun et al., 2013; Xiao et al., 2013; Lu et al., 2015). Some scholars applied the neural network model to carbon emission prediction and analysis (Feng, 2011; Li, 2013), but there are few applications in the direct residents' living carbon emission, and the selected neural network model is single, which cannot strongly prove the superiority of the selection of prediction methods. It is to explore the possibility of the direct residents' carbon emissions in China reaching a peak in 2030 while selecting the optimal prediction model using different neural network model to predict the direct carbon emissions of China's residents, and which provide a certain theoretical basis for the researchers.

\footnotetext{
* Corresponding author
} 


\section{DATA AND METHODS}

\subsection{Direct carbon emissions and accounting}

Direct carbon emissions are generally refers to the carbon emissions generated by residents in the direct consumption of energy, which may generate by residents' basic living energy (including coal, gas, and electricity, etc.) such as cooking, household appliances, and heating (Ye et al., 2010; Deng et al., 2014). The study takes the common provincial administrative units as the scale (Ghosh et al., 2010; Lu et al., 2014), given a reference to "2006 IPCC Guidelines for National Greenhouse Gas Inventories"(IPCC, 2006), and uses the "bottom-up" carbon emissions coefficient method to calculate the per capita direct carbon emissions ( $t / t e n$ thousand people) of the entire China and its 30 provinces respectively from 1995 to 2014(Zhang et al., 2012; Baiocchi et al., 2015). The carbon emission coefficient method expresses as formula (1):

$$
\mathrm{C}_{\mathrm{i} j}=\mathrm{E}_{\mathrm{i} j} \cdot \mathrm{EF}_{\mathrm{j}}
$$

Where: $C_{i j}$ is the carbon emissions of the jth fuel in the ith region; $\mathrm{E}_{\mathrm{ij}}$ is the terminal consumption of the jth fuel in the ith region, the data comes from the provincial energy balance sheets of the China Energy Statistical Yearbook, $j=1,2,3 \ldots 19$, representing 19 types of energy such as coal, oil, natural gas, thermal power, and electricity; $\mathrm{EF}_{\mathrm{j}}$ is the $\mathrm{CO} 2$ emission coefficient of the jth fuel, originated from the "2006 IPCC Guidelines for National Greenhouse Gas Inventories"; the carbon emission coefficient of each province and city is derived from the "2014 Baseline Emission Factor of China's Regional Power Grids"; total population as well as urban and rural population is from the China Statistical Yearbook 1995-2014.

It should be pointed out that, due to the lack of relevant statistical data, the research area does not include the carbon emission data of four regions, including the Tibet, Taiwan, Hong Kong, and the Macao Special Administrative Region. Furthermore, the relevant data of Chongqing in 1995 and 1996 and the relevant data of Ningxia from 2000 to 2002 are missing, and the moving average method is adopted to supplement these to ensure the continuity of data.

\subsection{Neural network model}

As a branch of artificial intelligence discipline, artificial neural network has a strong ability of nonlinear processing and is widely used in pattern recognition, nonlinear fitting, problem optimization, data analysis and prediction. And the neural network models of BP, RBF and Elman all have important applications in the field of prediction.
2.2.1 BP neural network design: BP (Back Propagation) neural network consists of input layer, hidden layer and output layer, which is a widely used multi-layer feedforward neural network model. This study divided the test data into 16 data samples, that is, the carbon emission data of each successive 4 years from 1995 is used as the data input, and the carbon emission data of the fifth year is used as the data output. Since a three-layer BP neural network with only one hidden layer can perform any m-dimensional to n-dimensional function mapping (Liu, 2013), and in order to facilitate compared with RBF, a three-layer BP neural network is designed for this study. Combining with Kolmogorov's theorem, after several experiments (Wu, 1999), to select logarithmic activation function and linear transfer function with better performance as transfer function, and to determine that the number of hidden layer neurons in the model is 19 , the output layer node number is 1 , it adopts the adaptive learning rate momentum gradient traingdx algorithm with fast convergence speed and high training accuracy to predict the carbon emission data of the test regions in 2013 and 2014 and output the results, and selects the residual, mean absolute Errors (MAE) and mean squared errors (MSE) as a measure of prediction accuracy.

2.2.2 RBF neural network design: The RBF (Radial Basis Function) neural network is a three-layer feed-forward neural network model with only one hidden layer. Different from the BP neural network design, during the network testing, its design method is that all the provincial carbon emissions data will be based on the data of the $20 \times 31$ matrix, and the test area data is used as the test sample, the remaining 30 columns of data are served as training samples, meanwhile, the data of the first 18 years are used as input samples, and the data of year 2013 and 2014 are used as the expected output samples. In addition, in order to make full use of the sample data in the process of prediction and improve the prediction accuracy and the generalization ability of the network, the interpolation method with the default value of the linear spline function is adopted to perform two-dimensional cubic interpolation on the sample data, which increase the number of learning samples to 100 . Then, the newrb function is used to create a regularized RBF neural network model, and the same error evaluation factor as the BP neural network is taken as the accuracy evaluation index.

2.2.3 Elman neural network design: Elman neural network is a dynamic feedback neural network model with local memory and feedback capability, has good convergence speed and high prediction accuracy, and it is mostly used to solve the problem of time series prediction in real applications. In contrast to the above two neural networks, the Elman neural network has added a transition layer with memory and feedback functions in addition to the conventional input layer, hidden layer and output layer. The layer can be regarded as a set of delay operators, and the output of the last moment in the memory hidden layer is used as the output of the next moment, which transmitting the data back to the hidden layer so that the network has a dynamic memory and feedback function. Similarly, the Elman neural network design adopts the same design parameters as the BP neural network as well as selects the same error evaluation index. 


\section{RESULTS AND ANALYSIS}

\subsection{Carbon emissions and accounting}

Using the carbon emission coefficient method, the carbon emissions data of 30 provinces and China as a whole are obtained (Figure1). As can be seen from the figure, the per capita direct carbon emissions of China's provinces and cities have increased in different ranges over the past 20 years. Of which, Beijing and Tianjin have always been the regions with high direct carbon emissions of Chinese residents, and reaching approximately $2.0514 \mathrm{t}$ and $1.9504 \mathrm{t}$ per ten thousand person respectively in the year of 2014; On the contrary, Hainan and Guangxi is relatively low, reaching $0.4630 \mathrm{t}$ and $0.4559 \mathrm{t}$ per ten thousand person respectively in 2014 , and the direct carbon emissions in most parts of the country have shown a significant growth trend since 2005 . It should be noted that the abnormally high value in Inner Mongolia during the period 2010-2012, the main reason is that coal consumption is accounted for a major part of the energy consumption structure in the region, and there is a decline in 2013 after the adjustment of energy consumption structure.

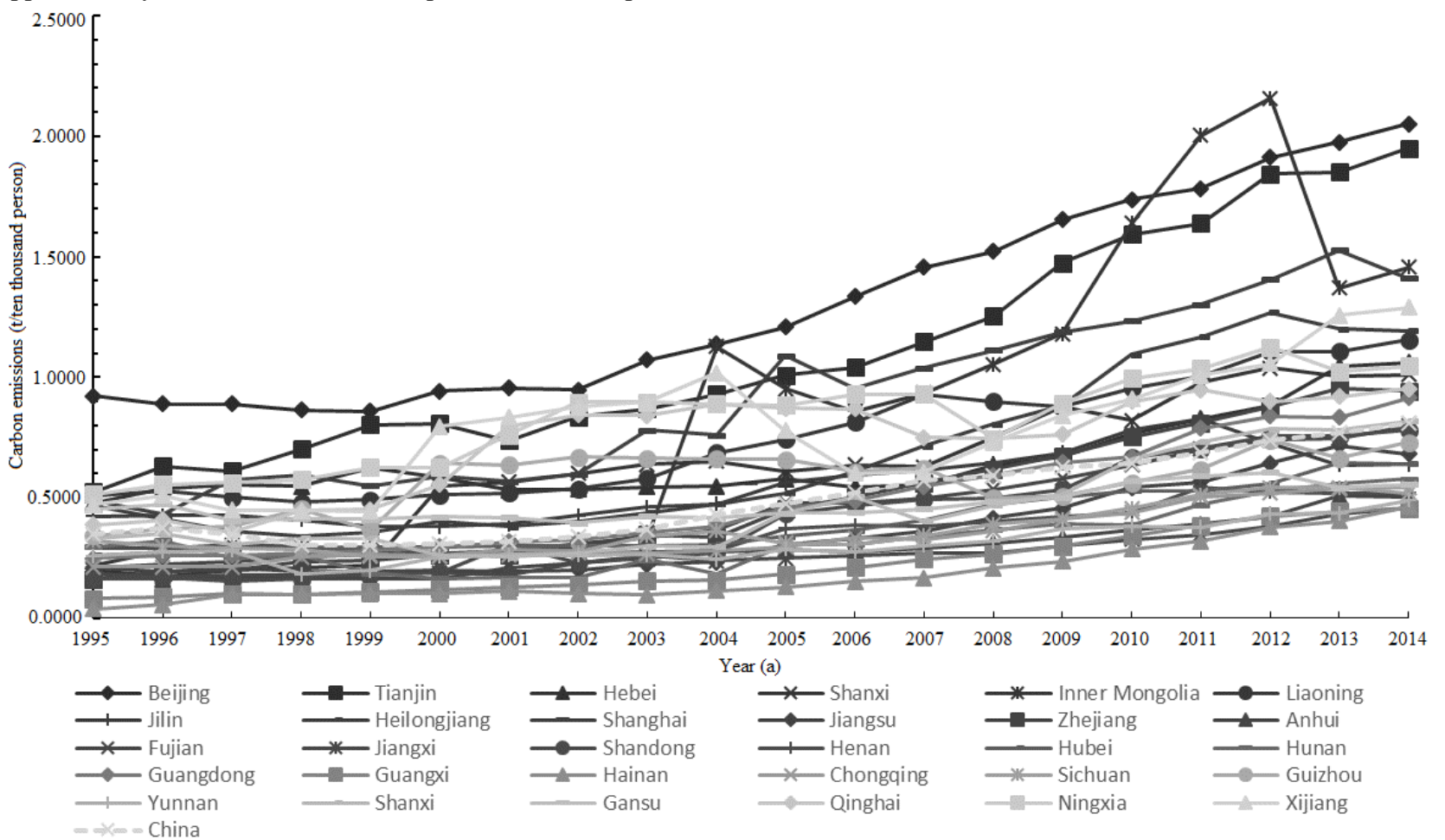

Figure 1. Residents' direct carbon emissions of China and provinces from 1995 to 2014

\subsection{Comparison of models}

For a scientific and reasonable selection result, this paper selects Beijing with the highest per capita carbon emission, Henan Province with the largest population base, and Hainan Province with the lowest per capita carbon emissions as the prediction test data, and uses three different neural network models to predict the direct residents' carbon emission of them, the comparison between real and predicted value, MAE, MSE and its average value are selected as the evaluation indicators from the results. The prediction results are shown in Table 1 .

\begin{tabular}{|c|c|c|c|c|c|c|c|c|c|c|}
\hline \multirow{2}{*}{\multicolumn{2}{|c|}{ Project }} & \multicolumn{3}{|c|}{2013} & \multicolumn{3}{|c|}{2014} & \multirow{2}{*}{ MAE } & \multirow{2}{*}{$\begin{array}{c}\text { MSE } \\
\left(10^{-2}\right)\end{array}$} & \multirow{2}{*}{$\begin{array}{c}\text { Average } \\
\text { MSE } \\
\left(10^{-2}\right)\end{array}$} \\
\hline & & $\begin{array}{c}\text { Real } \\
\text { value }\end{array}$ & $\begin{array}{l}\text { Predicted } \\
\text { value }\end{array}$ & $\begin{array}{l}\text { Error } \\
\text { value }\end{array}$ & $\begin{array}{c}\text { Real } \\
\text { value }\end{array}$ & $\begin{array}{c}\text { Predicted } \\
\text { value }\end{array}$ & $\begin{array}{l}\text { Error } \\
\text { value }\end{array}$ & & & \\
\hline \multirow{3}{*}{$\mathrm{BP}$} & Beijing & 1.9754 & 1.9882 & 0.0128 & 2.0514 & 2.0419 & -0.0095 & \multirow{3}{*}{0.0181} & 0.0127 & \multirow{3}{*}{0.0395} \\
\hline & Henan & 0.5162 & 0.4857 & -0.0305 & 0.5199 & 0.5331 & 0.0132 & & 0.0554 & \\
\hline & Hainan & 0.4042 & 0.3898 & -0.0144 & 0.4630 & 0.4913 & 0.0283 & & 0.0504 & \\
\hline \multirow{3}{*}{$\mathrm{RBF}$} & Beijing & 1.9754 & 1.9854 & -0.0100 & 2.0514 & 2.1542 & -0.1028 & \multirow{3}{*}{0.0447} & 0.5338 & \multirow{3}{*}{0.3080} \\
\hline & Henan & 0.5162 & 0.4563 & -0.0599 & 0.5199 & 0.5479 & 0.0280 & & 0.2188 & \\
\hline & Hainan & 0.4042 & 0.3948 & -0.0094 & 0.4630 & 0.4052 & -0.0578 & & 0.1715 & \\
\hline \multirow{3}{*}{ Elman } & Beijing & 1.9754 & 1.9796 & 0.0042 & 2.0514 & 2.0364 & -0.0149 & \multirow{3}{*}{0.0093} & 0.0121 & \multirow{3}{*}{0.0195} \\
\hline & Henan & 0.5162 & 0.5117 & -0.0045 & 0.5199 & 0.5274 & 0.0075 & & 0.3011 & \\
\hline & Hainan & 0.4042 & 0.4173 & 0.0131 & 0.4630 & 0.4746 & 0.0116 & & 0.0153 & \\
\hline
\end{tabular}

Table 1. Comparison of prediction accuracy of three neural network models 


\section{It can be seen from Table 1:}

(1) The prediction results are all satisfactory other than Beijing in 2014 using the RBF neural network, the prediction error reaches to $-0.1028 \mathrm{t}$ per ten thousand person. Compared with the BP neural network, the RBF neural network has a simple network structure, does not require repeated training, and has a fast convergence rate, however, the prediction accuracy is low, and the average absolute error reaches $0.447 \mathrm{t}$ per ten thousand person, the prediction accuracy is obviously inferior to the $\mathrm{BP}$ neural network, so the RBF neural network is not suitable for the prediction of the data in this study.

(2) The BP neural network and Elman neural network have high prediction accuracy for the direct carbon emission in the three places, which can theoretically be used to forecast carbon emission data. Nevertheless, the BP neural network must be multiple trained based on experience to determine the best network, and large prediction errors occur occasionally during the course of training, compared with Elman neural network, it has less robustness and more randomness, in addition, both the convergence speed and the prediction accuracy are inferior to the Elman neural network, above all, the Elman neural network is more superior in carbon emission prediction and is more suitable for the study of this problem.

(3) MSE is a relatively simple method to measure the average error, which can be used to measure the overall extent of change
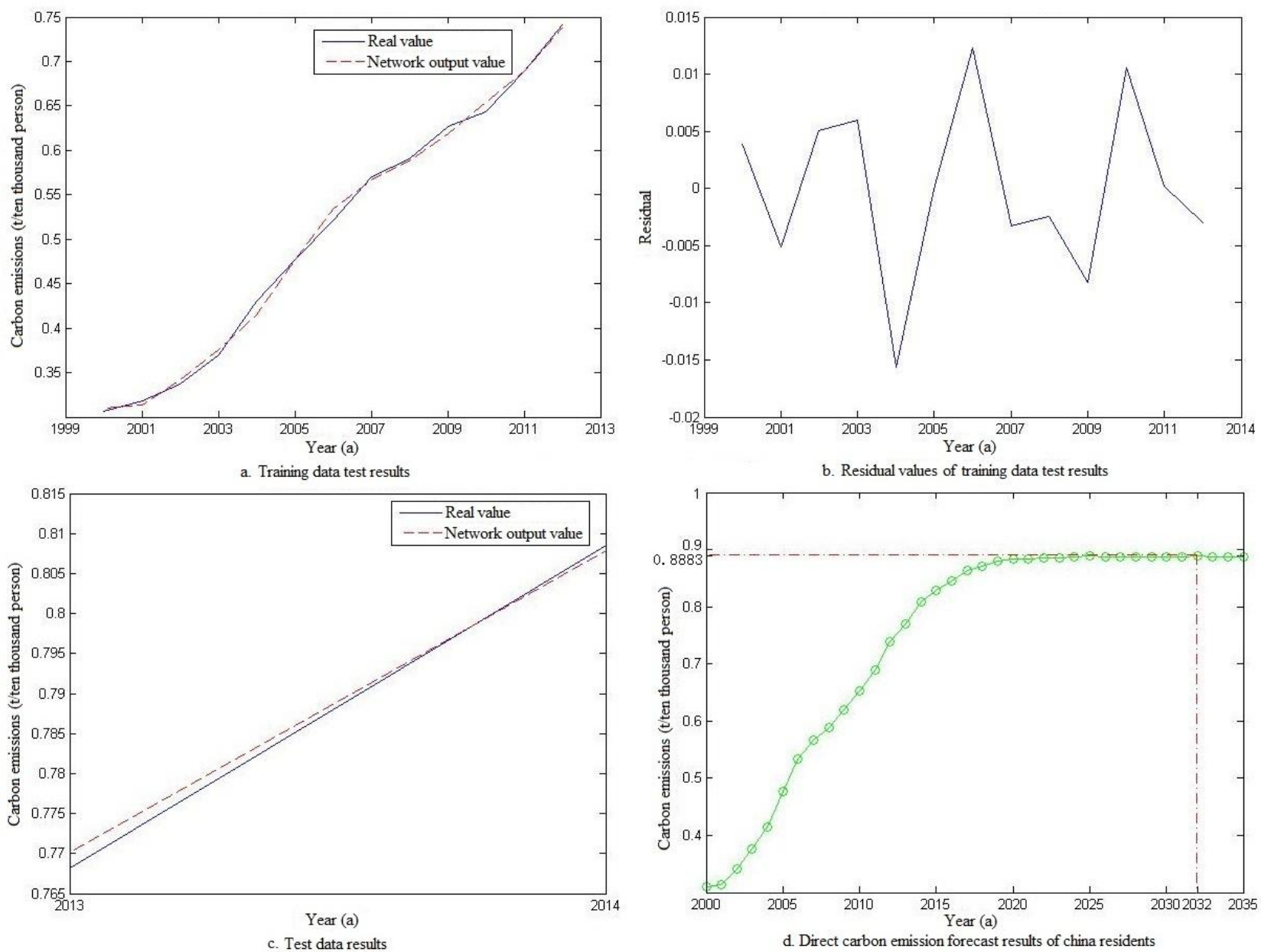

Figure 2. Forecast results of residents' direct carbon emissions in China 


\begin{tabular}{|c|c|c|c|c|c|c|c|c|}
\hline \multirow[b]{2}{*}{ Project } & \multicolumn{3}{|c|}{2013} & \multicolumn{3}{|c|}{2014} & \multirow[b]{2}{*}{ MAE } & \multirow{2}{*}{$\begin{array}{c}\text { MSE } \\
\left(10^{-2}\right)\end{array}$} \\
\hline & Real value & $\begin{array}{l}\text { Predicted } \\
\text { value }\end{array}$ & Error value & Real value & $\begin{array}{l}\text { Predicted } \\
\text { value }\end{array}$ & Error value & & \\
\hline Elman & 0.7682 & 0.7701 & 0.0019 & 0.8085 & 0.8079 & -0.0006 & 0.0013 & 0.0002 \\
\hline
\end{tabular}

Table 2. A comparison in prediction accuracy of residents' direct carbon emissions in China

From Figure 2, the a-graph shows that the test result of training data fits well with the real data, and b-graph shows that the residual value is basically between -0.015 and 0.01 , indicating that the network training is successful and effective. From cgraph and table 2, we can see that the test results are excellent for 2013 and 2014, and the predicted data is very accurate, the error values are 0.0019 and -0.0006 respectively, and MSE reaches $0.0002 \%$, which shows that the network prediction has an excellent performance and can be further used to predict the direct carbon emissions of the entire Chinese residents.

To predict carbon emissions in China around 2030, this study adds the cycles to the existing model parameters, thus, the direct carbon emission trends of Chinese residents from 2015 to 2035 are obtained, shown in Figure 2, the d-graph. The detailed carbon emission forecast data is listed in the Table 3.

\begin{tabular}{cccccccc}
\hline Year (a) & \multicolumn{7}{c}{ Carbon emissions (t/ten thousand person) } \\
\hline $2015-2021$ & 0.8280 & 0.8456 & 0.8642 & 0.8712 & 0.8808 & 0.8829 & 0.8831 \\
$2022-2028$ & 0.8855 & 0.8852 & 0.8873 & 0.8882 & 0.8879 & 0.8882 & 0.8874 \\
$2029-2035$ & 0.8876 & 0.8879 & 0.8880 & 0.8883 & 0.8881 & 0.8879 & 0.8879 \\
\hline
\end{tabular}

Table 3. Forecast results of residents' direct carbon emissions in China from 2015 to 2035

The d-graph in Figure 2 intuitively reflects the development trend of residents' direct carbon emissions in China to 2035 without considering any influencing factors and the current situation of free emission. Combining with Table 3, it can be seen that the per capita direct carbon emissions of Chinese residents will still show a relatively rapid growth trend within 5 years, and will gradually stabilize after 2020, then, after reaching the peak in 2032, the trend shows a slight decline with a peak of $0.8883 \mathrm{t}$ per ten thousand people. Based on the research and the analysis of this paper, at least in theory, China is expected to reach the peak of carbon emissions by 2030 .

\section{CONCLUSION AND DISCUSSION}

Based on the accounting for the residents' direct carbon emission data of Chinese province from 1995 to 2014, the paper uses BP, RBF, and Elman neural networks to predict the direct residents' carbon emissions in Beijing, Henan, and Hainan, respectively, and also uses the Elman neural network to predict the direct residents' carbon emission in China around 2030. The results show that:

(1) The per capita direct carbon emissions of China's provinces and cities have shown an increasing trend in different ranges over the past 20 years. Among them, Beijing and Tianjin have always been the regions with high direct carbon emissions of Chinese residents, and reached approximately $2.0514 \mathrm{t}$ per ten thousand person and $1.9504 \mathrm{t}$ per ten thousand person respectively in the year of 2014; the per capita direct carbon emissions in most regions of the country have shown a clear upward trend since 2005.

(2) The RBF neural network model is superior to the other two networks in terms of network structure, number of iterations, and convergence rate, but the error is larger than them; both BP and Elman neural networks have high prediction accuracy, and their MSE average are $0.0395 \%$ and $0.0195 \%$ respectively, which can be used for the prediction of direct carbon emission data theoretically, however, BP neural network model is slightly more tedious in the process of construction and learning, the robustness and fault tolerance are poor, and its prediction accuracy is slightly inferior to the Elman neural network. Therefore, Elman's neural network has the best prediction performance, which is more suitable for the prediction of direct residents' carbon emissions.

(3) Without any external force and free development of carbon emissions, the residents' direct carbon emissions in China will show an increasing trend from fast to slow in the next few years. The per capita residents' carbon emissions will decline slightly after peaking in 2032, peak of $0.8883 \mathrm{t}$ per ten thousand person. In theory, China's emission is expected to reach its peak around 2030.

This study is aimed at predicting and analyzing the direct carbon emissions of China's provincial residents, and thus its development trend is obtained, which confirmed the feasibility of Elman neural network as well as provided the researchers with a certain theoretical basis. However, the availability and accuracy of statistical data have affected the accuracy of prediction results in a certain extent, and carbon emissions are actually affected by many factors, and residents' direct carbon emission is only a relatively important part of the total carbon emissions. Thus, forecast and analysis of total carbon emissions under the influence of multiple factors will be the focus of the next step research.

\section{ACKNOWLEDGEMENTS}

Acknowledgements of support for the Key Scientific Research Projects in Colleges and Universities (17A170005); China Post doctoral fund (2016M602232). The National Natural Science F und $(41675029,41671425,41565008)$. Open Fund of Henan K ey Laboratory of Integrated Air Pollution Control and Ecologica 1 Security (20170201). 


\section{REFERENCES}

Baiocchi G, Creutzig F, Minx J, et al., 2015. A spatial typology of human settlements and their $\mathrm{CO} 2$, emissions in England. Global Environmental Change, pp.13-21.

Druckman A, Jackson T, 2009. The carbon footprint of UK households 1990-2004: a socio-economically disaggregated, quasi-multi-regional input-output model. Ecological Economics, 68(7), pp.2066-2077.

Deng J, Liu X, Wang Z, 2014. Characteristics analysis and factor decomposition based on the regional difference changes in China's CO2 emission. Journal of Natural Resources, 2014, 29(2), pp. 189-200.

Fang J Y, Zhu J L, Wang S P, et al., 2011. Global warming, human-induced carbon emissions, and their uncertainties. Science China Earth Sciences, 54(10), pp. 1458-1468.

Feng R, 2011. Study on the methods and application of estimation of $\mathrm{C} 02$ emission on urban household energy consumption. NanKai University.

Ghosh T, Elvidge C D, Sutton P C, et al., 2010. Creating a Global Grid of Distributed Fossil Fuel CO2 Emissions from Nighttime Satellite Imagery. Energies, 3(12), pp. 1895-1913.

Huang R, Wang Z, Ding G, et al., 2016. Trend prediction and analysis of influencing factors of carbon emissions from energy consumption in Jiangsu province based on STIRPAT model. Geographical Research, 35(4), pp. 781-789.

IPCC, 2006. 2006 IPCC guidelines for national greenhouse gas inventories. IGS, Japan: The National Greenhouse Gas Inventories Programme.

Lu H, Liu G, 2014. Spatial effects of carbon dioxide emissions from residential energy consumption: A county-level study using enhanced nocturnal lighting. Applied Energy, 131(9), pp. 297-306.

Li Y M, Yang T, 2013. Comparison of Urban and Rural Household Direct Energy Consumption and CO_2 Emissions. Resources Science, 35(1), pp. 115-124.

Lean J L, Rind D H, 2008. How natural and anthropogenic influences alter global and regional surface temperatures: 1889 to 2006. Geophysical Research Letters, 35(18), pp. 102-102.

Lu Z H, Wu X D, Guo L B, et al., 2015. Prediction of the Number of Rodent Community Composition Species Based on Elman Neural Network in Alasan Desert. Ecology \& Environmental Sciences, (12), pp. 1976-1982.

Luo X, Niu H Q, Lin H R, et al., 2013. Application and contrast analysis of $\mathrm{BP}$ and $\mathrm{RBF}$ neural network in prediction of breakdown voltage of air gap. Advanced Technology of Electrical Engineering \& Energy, 32(3), pp. 110-115.

Qin D H, 2006. China's climate and environmental changes and their impacts and countermeasures. Theoretical Trends, pp. 2838 .

Qin Y C, 2013. The Model and Method of Low-carbon City Research. Science Press, pp.10-21.
Qu S N, Guo C X, 2010. Forecast of China's carbon emissions based on STIRPAT model. China Population Resources \& Environment, 20(12), pp. 10-15.

Shi A. 2003. The impact of population pressure on global carbon dioxide emissions, 1975-1996: evidence from pooled cross-country data. Ecological Economics, 44(1), pp. 29-42.

Shui B, Dowlatabadi H, 2005. Consumer lifestyle approach to US energy use and the related CO2, emissions. Energy Policy, 33(2), pp. 197-208.

Sun J F, Geng Y L, Guo Y R, et al., 2013. Application and comparison of Elman neural network model and ARIMA model to predict the incidence of influenza. Journal of Zhengzhou University, (5), pp. 584-587.

Wu C X, Liu L, Wang B Y, 1999. The Study of the Method to Determining the Number of Hidden Units of Three layer BP Neural Networks. Journal of Wuhan Technical University of Surveying \& Mapping, 24(2), pp. 177-179.

Wang S W, 2010. The global warming debate. Science Bulletin, 55(19), pp. 1961-1962.

Xiao J C, Ou W X, Fu H Y, 2013. Land cover classification of Yancheng Coastal Natural Wetlands based on BP neural network and ETM+ remote sensing data. Acta Ecologica Sinica, 33(23), pp. 7496-7504.

Ye H, Pan L Y, Chen F, et al., 2010. Direct carbon emission from urban residential energy consumption: a case study of Xiamen, China. Acta Ecologica Sinica, 30(14), pp. 3802-3811.

Zhang Y, Qin Y C, Yan W Y, et al., 2012. Urban types and impact factors on carbon emissions from direct energy consumption of residents in China. Geographical Research, 13(3), pp. 409-417.

Zhao X, Qi J M, Liu G W, 2013. China carbon emission forecast based on the Discrete Difference Equation Prediction Model. Journal of Arid Land Resources \& Environment, 27(1), pp. 63-69.

Zhu Y B, Wang Z, Pang L, et al., 2009. Simulation on China"s Economy and Prediction on Energy Consumption and Carbon Emission under Optimal Growth Path. Acta Geographica Sinica, 64(8), pp. 935-944

Zhuang G Y, 2009. Copenhagen Climate Game and the Recognition of China's Role. Foreign Policy Review, 26(6), pp. 13-21. 